\title{
Detector Based Calibration of a Portable Imaging Spectrometer for CLARREO Pathfinder Mission
}

\author{
Yigit Aytac $^{\mathrm{a}}$, Kurt Thome ${ }^{\mathrm{b}}$, Brian N. Wenny ${ }^{\mathrm{a}}$, Amit Angal ${ }^{\mathrm{a}}$, Timothy M. Shuman ${ }^{\mathrm{c}}$, and \\ Brendan McAndrew ${ }^{\mathrm{b}}$

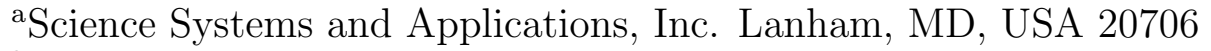 \\ ${ }^{\mathrm{b}}$ NASA Goddard Space Flight Center, Greenbelt, MD, USA 20771 \\ ${ }^{\mathrm{c}}$ FiberTech, Inc., Herndon, VA, USA 20171
}

\begin{abstract}
The Climate Absolute Refractivity and Reflectance Observatory (CLARREO) Pathfinder (CPF) mission is being developed to demonstrate SI-traceable retrievals of reflectance at unprecedented accuracies for global satellite observations. An Independent Calibration of the CPF sensor using the Goddard Laser for Absolute Measurement of Radiance (GLAMR) is planned to allow validation of CPF accuracies. GLAMR is a detector-based calibration system relies on a set of NIST-calibrated transfer radiometers to assess the spectral radiance from the GLAMR sphere source to better than $0.3 \%(\mathrm{k}=2)$. The current work describes the calibration of the Solar, Lunar Absolute Reflectance Imaging Spectroradiometer (SOLARIS) that was originally developed as a calibration demonstration system for the CLARREO mission and is now being used to assess the independent calibration being developed for CPF. The methodology for the radiometric calibration of SOLARIS is presented as well as results from the GLAMR-based calibration of SOLARIS. The portability of SOLARIS makes it capable of collecting field measurements of earth scenes and direct solar and lunar irradiance similar to those expected during the on-orbit operation of the CPF sensor. Results of SOLARIS field measurements are presented. The use of SOLARIS in this effort also allows the testing protocols for GLAMR to be improved and the field measurements by SOLARIS build confidence in the error budget for GLAMR calibrations. Results are compared to accepted solar irradiance models to demonstrate accuracy values giving confidence in the error budget for the CLARREO reflectance retrieval.
\end{abstract}

Keywords: Radiometry, Visibility and imaging, Photodetectors, Image analysis

\section{INTRODUCTION}

The Climate Absolute Refractivity and Reflectance Observatory CLARREO Pathfinder (CPF) project is a risk reduction mission to demonstrate the capability to make reflected solar measurements suitable for a full CLARREO Mission. CLARREO was a Tier-1 Decadal Survey mission that was to provide earth observation data to understand uncertainties in climate change models. ${ }^{1,2}$ At the core of the CLARREO project were four fundamental goals: i) Achieving traceability to SI (système international) standards, ii) obtaining temporal and angular sampling to detect decadal climate change signals above natural variability, iii) collecting sufficient information content to infer climate change in key variables of interest, and iv) demonstrating inter-calibration capabilities. The accuracy requirements to observe climate change to within the uncertainty of the limit of natural variability are shown to be $0.1 \mathrm{~K}(\mathrm{k}=3)$ for the infrared spectrum, and $0.3 \%(\mathrm{k}=2)$ in absolute reflectance for the solar spectrum. CPF has two primary mission objectives: demonstrate on orbit, high accuracy, SI-Traceable calibration in the reflected solar and demonstrate an ability to transfer this calibration to other on-orbit assets. The CLARREO Pathfinder is planned to be mounted on the International Space Station for a nominal one year mission life, followed by an additional year for science data analysis. The mission is a Category 3 / Class D with a current launch readiness date of 2023 . The absolute radiometric uncertainty of CPF is less than $0.3 \%(\mathrm{k}=1)$.

Demonstrating the stringent radiometric uncertainty of CPF is a difficult task since the CPF required uncertainty represents an improvement of a factor of five over the current state of the art, on-orbit earth missions.

Yigit Aytac: E-mail: yigit.aytac@nasa.gov 
Achieving the required uncertainty depends on advancements in both instrument design and on-orbit calibration techniques. Demonstrating the on-orbit radiometric uncertainty of CPF depends upon an Independent Calibration approach that relies on an independent, pre-launch absolute radiometric calibration of the CPF sensor. An instrument-model is used to transfer the laboratory calibration to orbit relying on direct solar irradiance measurements to evaluate the sensors behavior on orbit.

The absolute radiometric calibration for the Independent Calibration will rely on the Goddard Laser for Absolute Measurement of Radiance (GLAMR) that is a highly versatile optical characterization test unit using detector-based standards and tunable monochromatic lasers. ${ }^{3}$ It can be considered as a portable version of NISTs Spectral Irradiance and Radiance Responsivity Calibrations Using Uniform Sources (SIRCUS). ${ }^{4-7}$ GLAMR has already been used to characterize the radiometry of multiple sensors ${ }^{8-10}$ but not at the level of uncertainty needed for the CPF Independent Calibration.

One of the key efforts for the Independent Calibration is to assess and validate the uncertainty of the approach and evaluate the approach and accuracy for a GLAMR-based calibration. The SOlar, Lunar for Absolute Reflectance Imaging Spectroradiometer (SOLARIS) is being used to evaluate the Independent Calibration process. SOLARIS was designed to achieve the same performance specifications as the original CLARREO Reflected Solar instrument. Optical characterization of SOLARIS has been carried out using GLAMR to determine an absolute spectral response for the instrument. The results from those calibrations are presented in the current work as well as descriptions of both experimental methodology and processing algorithms that were applied to the SOLARIS calibration.

One element of the CPF Independent Calibration is validation of the laboratory calibration using on-orbit measurements. The portability of the SOLARIS instrument allows outdoor measurements of direct solar irradiance and solar-illuminated diffuser data. ${ }^{9,11}$ The solar spectral shape and irradiance provide a reliable source for on-orbit characterization and calibration. The SOLARIS field measurements also provide test data sets that will be used to develop the processing methods and algorithms for on-orbit CPF measurements. Field measurements with SOLARIS performed at NASA Goddard Space Flight Center on July 10, 2019 are presented along with comparison to results obtained from a non-imaging field-spectrometer.

\section{EXPERIMENTAL SETUP}

Laboratory characterization of SOLARIS has included absolute radiometric calibration using extended radiance sources as well as the geometric factors needed to convert the earth scene data to reflectance. ${ }^{12}$ This section describes the SOLARIS instrument concentrating on those aspects of the sensor that are of direct relevance to the GLAMR calibrations. Details on the GLAMR system as it applies to the calibration of SOLARIS, and eventually the CPF flight instrument are provided including approaches developed to optimize the synchronization of the imaging spectrometer data and GLAMR transfer radiometers.

\subsection{SOLARIS}

SOLARIS was designed and assembled at GSFC as a calibration demonstration system for the original CLARREO mission. ${ }^{13}$ SOLARIS is an Offner push-broom style imaging spectro-radiometer that covers the visible to near-IR spectral region. ${ }^{14}$ The focal plane is composed of an off-the-shelf, silicon-based sCMOS detector with 21602560 pixels and 16-bit A/D. The detector is sensitive over the $350 \mathrm{~nm}$ to $1000 \mathrm{~nm}$ spectral range. The polarization sensitivity of SOLARIS is less than $0.5 \%$ through the use of dual quartz depolarizes. Neutral density (ND) filters are used to attenuate the direct solar irradiance both to avoid damaging the sCMOS detector and to provide energy levels similar to unattenuated earth science measurements.

Past efforts as part of the CLARREO project performed component-level tests of the SOLARIS optics, detectors, grating, depolarizers and attenuators. ${ }^{8,9}$ Past work has also determined the SOLARIS relative spectral response (RSR), detector- to-detector uniformity, noise, and temperature sensitivity. In addition, the measurements that related to the geometrical factor of instrument such as pixel pitch and orientation of array relative to fiducial were determined for a previous version of the detector package. The grating performance and dimensional metrology was also tested, and the alignment of the sCMOS detector established an orthogonal spatial and spectral axis. 

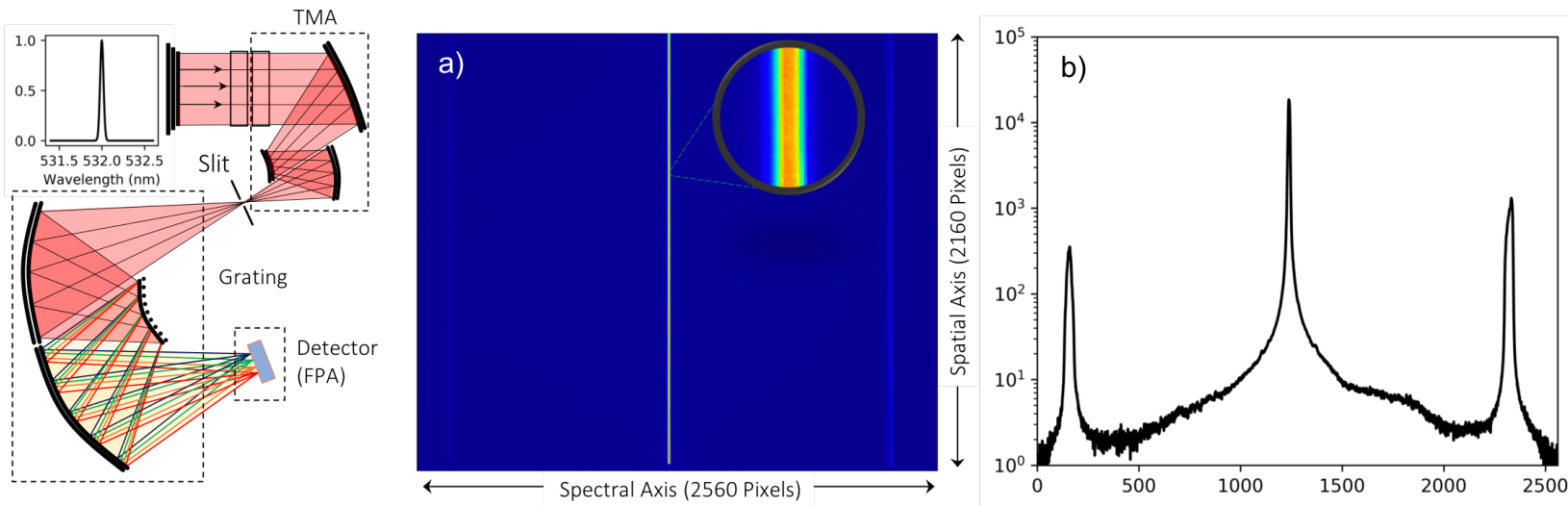

Figure 1. Geometry of an Offner spectrometer is shown on left. (a) A SOLARIS image of the GLAMR integrating sphere is shown at $532 \mathrm{~nm}$ wavelength. (b) Image profile of the SOLARIS image is shown as a function of spectral pixel numbers.

A representative frame from SOLARIS while viewing the GLAMR integrating sphere is shown in Figure 2 (a), and the image profile in Digital Number per seconds (DN/s) as a function of the spectral pixel number is shown in Figure 2 (b). The horizontal axis of the image contains the spectral information where the first vertical line from right is the 0 th order diffraction which marks $0 \mathrm{~nm}$. The second vertical line from right side of the image is the (-) 1st order grating diffraction that signifies the spectral response of instrument. The wavelength associated with the center of each spectral pixel of SOLARIS is determined based on the location on the FPA of 0th and the (-) 1st order signals, the known GLAMR wavelength, and assuming a linear dispersion. The conversion constant from pixel to wavelength is determined to be $0.52 \mathrm{~nm} /$ pixel.

Another feature that is noticeable in Figure 1 is the how a monochromatic light source provides a information on higher order diffraction effects in a grating-based imaging spectrometer such as SOLARIS. The shape of the narrow vertical signal line in Figure 1 (b) and (c) is the combination of the slit, pixel, and grating functions which is approximately $9 \mathrm{~nm}$ or 18 pixels in full-width half-maximum (FWHM) intensity. Changes in the width or line shape along the spatial dimension can indicate optics-related artifacts; misalignment of the slit relative to the FPA and/or grating.

\subsection{GLAMR System}

As mentioned, GLAMR is a near-monochromatic and tuneable light source with SI-traceability that can provide information about the instruments stray light, spectral response, and polarization at the sensitivity levels necessary for the CLARREO Pathfinder mission. NISTs SIRCUS facility was the basis of the original GLAMR source and is still considered as a model for the updated GLAMR characterization system. In both systems, the Primary Optical Watt Radiometer (POWR), a high accuracy cryogenic radiometer operating by the electrical substitution method, determines the output of a source that is used to determine the absolute spectral response (ASR) of a transfer radiometer. The GLAMR SI-tracebility is to POWR via the calibration of the detector-based transfer radiometer used in the GLAMR facility. The source used in the process is the POWR laser which is a tunable, stabilized laser source allowing the ASR of the GLAMR transfer radiometer to be determined across the full spectral range of the transfer radiometer. The GLAMR system currently has a total of 15 transfer radiometers, of which there are five silicon-based trap detector radiometers for visible to NIR, five short range single InGaAs detector radiometers for the spectral range $<1600 \mathrm{~nm}$, and five extended range InGaAs sphere based detector radiometers for the spectral range $<2400 \mathrm{~nm}$. The transfer radiometers are used both within the GLAMR integrating sphere to provide an absolute measurement of the radiance during measurements of a sensor such as SOLARIS as well as externally to provide a transfer between the internal radiometers and a sensor under test.

The stated accuracy to calibrate a transfer radiometer in irradiance mode using POWR is $0.09 \%(\mathrm{k}=3)$. Recent work with the GLAMR facility indicates that the uncertainty the GLAMR sphere spectral radiance is 
$<0.57 \%(\mathrm{k}=2)$. Figure 2 shows an example of the measured spectral radiance from the GLAMR sphere across the SOLARIS spectral range. The spectral radiances shown in Figure 2 (a) are averages of five samples collected over approximately 30 seconds. Figure 2 (b) shows the standard deviation of the average for each of the data points shown in Figure 2 (a).
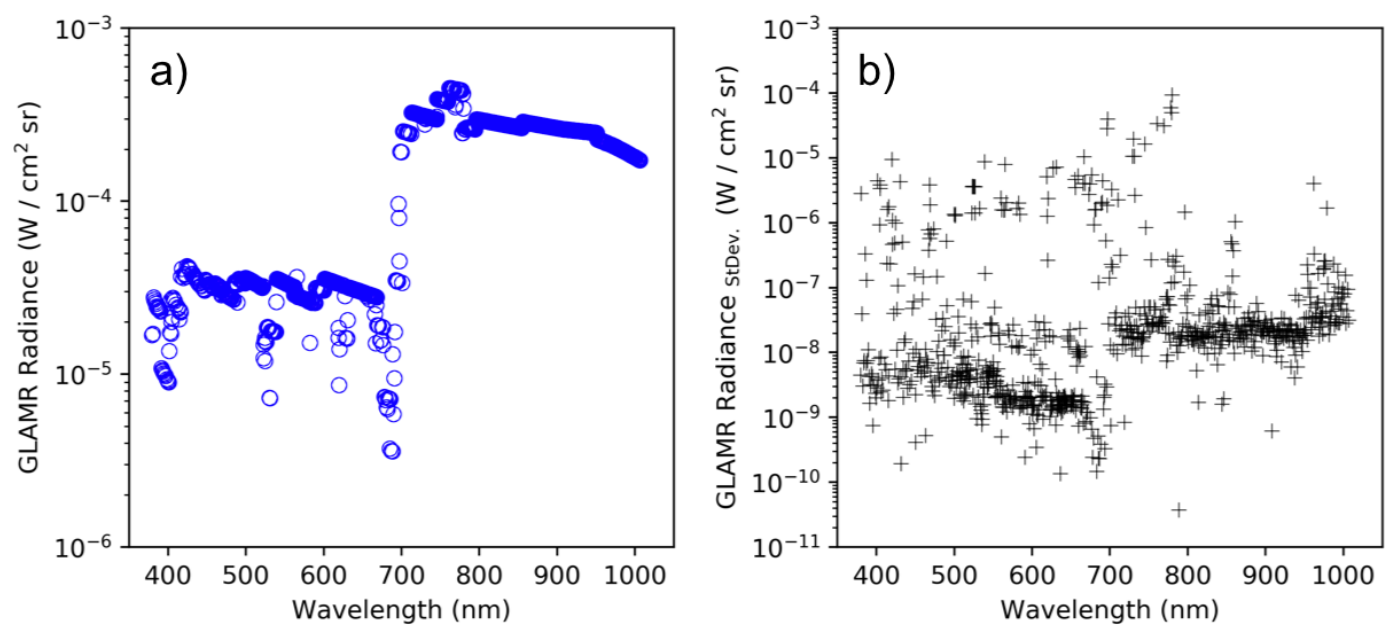

Figure 2. Geometry of an Offner spectrometer is shown on left. (a) A SOLARIS image of integrating sphere illuminated by GLAMR at $532 \mathrm{~nm}$. (b) Image profile of the $532 \mathrm{~nm}$ sphere image.

The GLAMR system used for the SOLARIS characterizations shown here rely on a frequency doubled neodymium doped yttrium vanadate at $532 \mathrm{~nm}$ operated at $80 \mathrm{MHz}$ repetition rate which is used to pump an optical parametric oscillator (OPO). The OPO produces tunable complementary signal and idler wavelengths with the fundamental tuning range being $680 \mathrm{~nm}$ to $1100 \mathrm{~nm}$, and the second harmonic generation (SHG) being $340 \mathrm{~nm}$ to $550 \mathrm{~nm}$. In addition, the mirrors become partially transmissive to the idler wavelength at $1200 \mathrm{~nm}$, so the idler output can be used from $1200 \mathrm{~nm}$ to $2400 \mathrm{~nm}$ with combined average powers in excess of several hundred milli-watts. Within each of these spectral ranges, wavelength tuning is fully automated by computer control of the lithium triborate crystal temperature and tuning prism stages. The monochromatic, broadband, and high-intensity output of the parametric conversion is coupled to a multi-mode fiber which is directed to the GLAMR integrating sphere.

\subsection{Data Acquisition}

The basic approach to the GLAMR calibration of SOLARIS begins by mounting the instrument so that its entrance aperture is centered on the exit port of the integrating sphere. The GLAMR source is tuned through the full spectral range with 30 SOLARIS frames collected for each tuned GLAMR wavelength. The GLAMR transfer radiometers monitor the sphere spectral radiance at a much higher sampling rate. The transfer radiometer measurements are used to determine the absolute spectral radiance incident on SOLARIS for each frame collected and these are used to determine the absolute spectral response of each detector in the FPA for each SOLARIS frame at each tuned GLAMR wavelength. The resulting spectral response data are used to derive the bandaveraged spectral response for each detector on the FPA as described below.

A master clock is used to link SOLARIS and GLAMR in time during the instrument characterization measurements. GLAMR transfer radiometer output as well as ancillary information such as transfer radiometer measurement time, source center wavelength, linewidth, and shutter configuration are recorded at a rate of $5 \mathrm{~Hz}$. The work presented here with SOLARIS only requires the VNIR trap detector transfer radiometers. Past work with the SOLARIS system has led to a sampling rate of $1 \mathrm{~Hz}$ based on the speed of the FPA A/D and signal to noise considerations. The timing diagram for the data acquisition is shown in Figure 3. The monitoring of the five transfer radiometer signals is set to measure the integrating sphere radiance five times while a wavemeter used to collect the GLAMR spectral information is synchronized with the GLAMR shutter opening. 


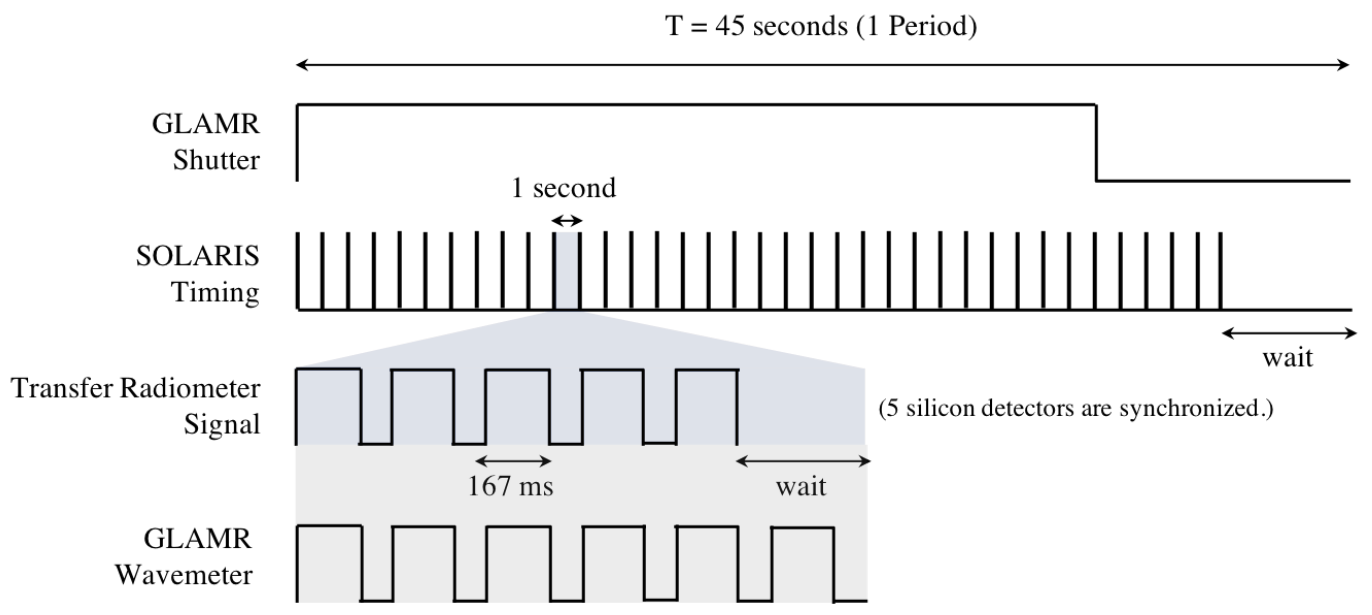

Figure 3. Timing diagram for the SOLARIS instrument calibration measurements. The waveforms are not to scale.

Thirty frames of SOLARIS data are illuminated by the GLAMR sphere are collected along with five dark SOLARIS frames over taking a period of 45 seconds at each tuned GLAMR wavelength. The captured SOLARIS images are stored in .tiff format requiring $10.8 \mathrm{Mb}$ of storage per image. The GLAMR system is tuned at a spectral sampling of $1 \mathrm{~nm}$ steps for most of the SOLARIS spectral range with the exception of the $500 \mathrm{~nm}$ to $675 \mathrm{~nm}$ spectral range which is sampled at $0.5 \mathrm{~nm}$ steps to enhance the GLAMR source performance. The integration time of SOLARIS is adjusted from $1 \mathrm{~ms}$ to $100 \mathrm{~ms}$ based on the GLAMR sphere radiance level.

\subsection{Data Processing}

The pre-processing of the SOLARIS data includes dark image subtraction of SOLARIS images. The GLAMR transfer radiometer data and ancillary data are re-sampled by averaging over $1 \mathrm{~Hz}$ to match the frame rate of SOLARIS data. The initial processing is to retrieve the absolute spectral responsivity (ASR) of the individual pixels at each tuned GLAMR wavelength. The ASR is defined as,

$$
A S R(\lambda, i, j)=D N(i, j) / \operatorname{Radiance}(\lambda),
$$

where $\lambda$ is the tuned GLAMR wavelength, $i$, and $j$ are the vertical and horizontal pixel numbers where $i$ refers to the spatial domain and $\mathrm{j}$ the spectral, respectively.

Measured SOLARIS signal and ASR values for 18 representative detectors are shown in Figure 4 (a) and (b), respectively over the $350 \mathrm{~nm}$ to $1000 \mathrm{~nm}$ wavelength range. Each data point is obtained from an individual SOLARIS frame as shown in Figure 1 (b). There are 30 images at each tuned GLAMR wavelength, therefore there are 30 unique SOLARIS signals and GLAMR radiance data points synchronized temporally. These data points share the same spectral information due to the temporal synchronization. The corresponding ASR value is calculated from the $\mathrm{DN} / \mathrm{s}$ and the GLAMR radiance values which is $2.42 \times 10^{10} \mathrm{DNs}^{-1} \mathrm{~W}^{-1} \mathrm{~cm}^{2} \mathrm{srnm}$. To be able to create clusters, density-based spatial clustering of applications with noise (DBSCAN) method is applied to the wavelength dependent ASR data for each pixel. A set of representative ASR curves for SOLARIS derived from a recent GLAMR calibration is shown in Figure 4 (b) for several SOLARIS detectors. This is repeated for all detectors in both the spatial and spectral domains.

Once the ASR for each detector is determined, the band averaged spectral response (R) is calculated ${ }^{15}$ as,

$$
R(\lambda, i, j)=\sum_{\lambda=350 n m}^{\lambda=1000 n m} \frac{A S R_{i, j}^{n}+A S R_{i, j}^{n-1}}{2} \times\left(\lambda^{n}-\lambda^{n-1}\right) .
$$

The center wavelength of the instrument is determined from, 


$$
\lambda_{i, j}^{\text {center }}=\frac{\sum \lambda A S R_{i, j}(\lambda) \Delta \lambda}{\sum A S R_{i, j}(\lambda) \Delta \lambda},
$$

where $\Delta \lambda$ is the difference between two consecutive measurement wavelengths. The expression in Equation 2 is the area under the ASR curve. Each of the 30 SOLARIS frames collected at a given tuned GLAMR wavelength are treated as unique data points in 2 rather than averaging the 30 and then computing $\mathrm{R}$.
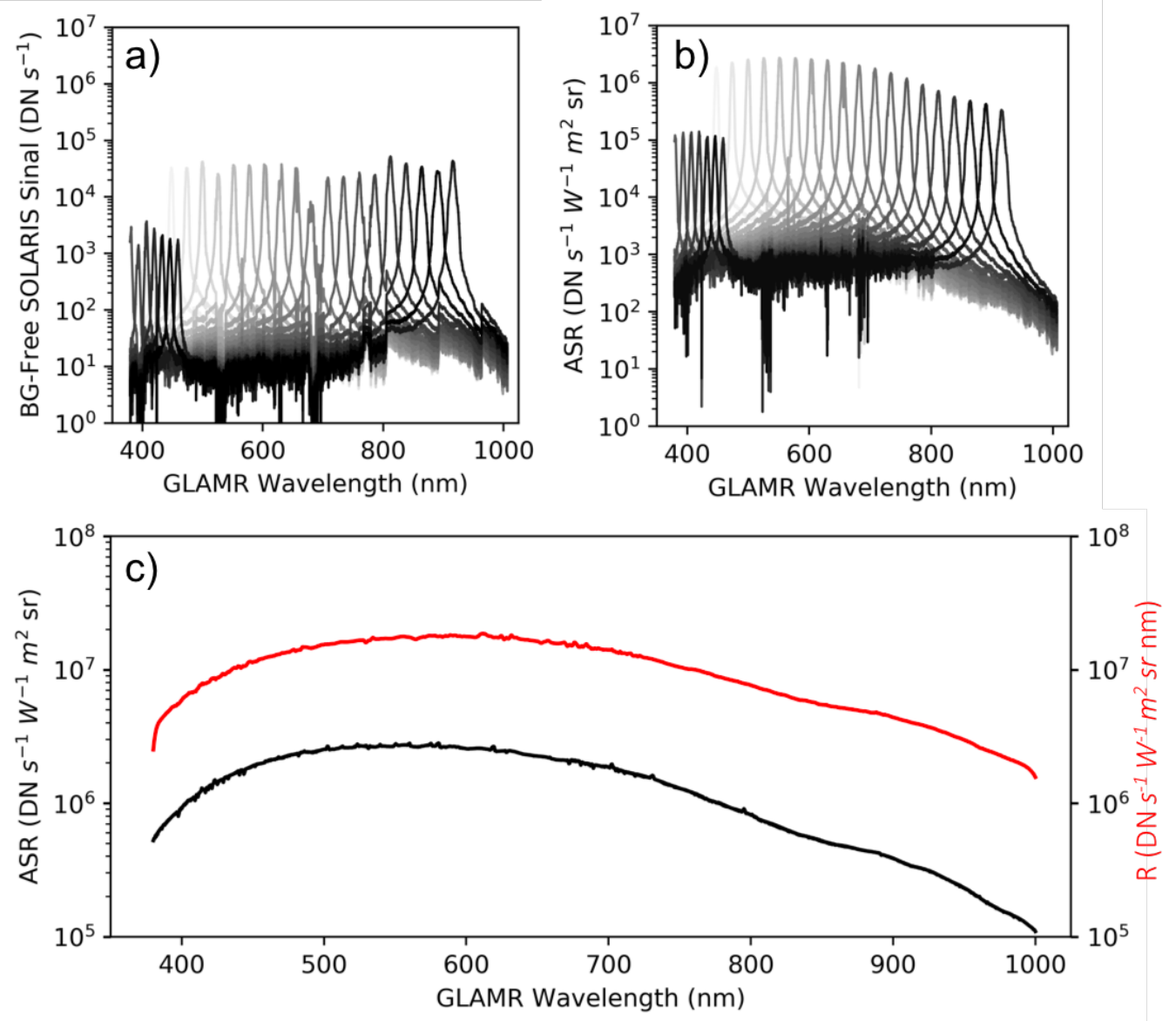

Figure 4. (a) The measured detector signal, (b) and the ASR values of SOLARIS is shown from $380 \mathrm{~nm}$ to $1000 \mathrm{~nm}$ spectral range. (c) The peak ASR value, and the calculated band averaged response of each pixel is shown as a function of tuned GLAMR wavelength.

\section{RESULTS}

The results of a recent GLAMR-based absolute calibration of SOLARIS are presented here. The results indicate that GLAMR provides a suitable calibration approach for an imaging spectrometer such as SOLARIS and, by extension, the CPF sensor. Further, the results for SOLARIS show that the instrument is a well-behaved sensor with reasonable focal plane uniformity, minimal stray light, and out-of-band response dominated by high-order grating effects. The results section also provides a discussion of the current understanding of uncertainties for the GLAMR absolute radiometric calibration. The development and testing of SOLARIS instrument leads to an end-to-end instrument performance model and credible error budget demonstrating the CLARREO Pathfinder required absolute uncertainties of $0.3 \%(\mathrm{k}=1)$ is achievable via GLAMR calibration procedure. Comparing the variability of the GLAMR and SOLARIS data sets individually is used to illustrate the probable sources of uncertainty in the calibration approach. The uncertainty sources for GLAMR testing can be split into three 
general terms: 1) uncertainty in the knowledge of the spectral radiance incident on the sensor under test; 2) synchronization of the GLAMR data with the instrument under test; 3) processing approaches deriving the absolute radiometric calibration. All three are discussed in this section.

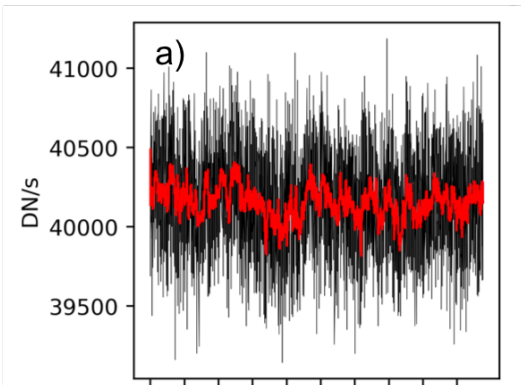

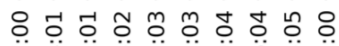

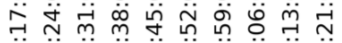

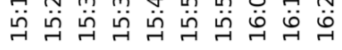
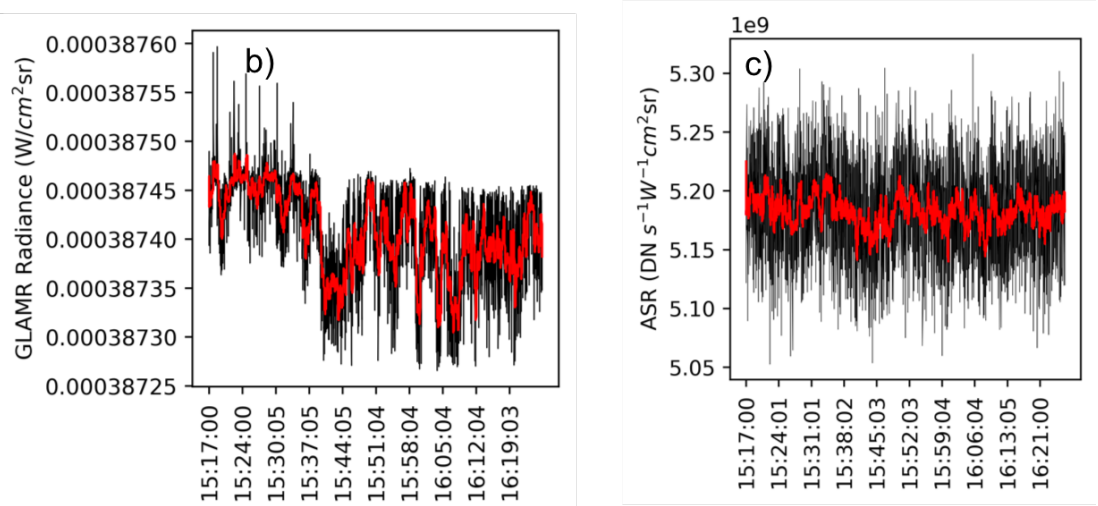

Figure 5. Long-term measurement results of SOLARIS response and GLAMR radiance are shown as a function of time. Each data point is obtained from an individual image, and GLAMR reading.

\subsection{SOLARIS Radiometric Calibration}

Band-averaged spectral response results from a recent GLAMR-based calibration are shown as the solid red line In Figure 4 (c). The results are shown for an average across 100 spatial rows of the SOLARIS FPA. One of the major advantages of a GLAMR-based calibration is that it provides an ASR for each detector while traditional source-based calibration approaches using broadband sources can only provide an approximation to the band-averaged spectral response. Traditional relative spectral response data can be scaled to an absolute output to approximate the ASR. The use of ASR in CPF processing will provide more accurate results than the band-averaged spectral response given a known source spectrum, but the band-averaged value provides a useful tool to compare the behavior of individual detectors relative to the others on the focal plane.

An additional metric used for the Independent Calibration is the peak ASR for each detector. Figure 4 (c) shows the peak ASR response as the black solid line. The peak ASR has a similar shape as the band averaged spectral response for much of the spectral range of SOLARIS. This indicates that the out-of-band response of the SOLARIS package is reasonably well behaved as would be expected with an imaging spectrometer.

It is noted that for wavelengths larger than $760 \mathrm{~nm}$ the shape of $\mathrm{R}$ begins to diverge from that of the peak ASR. The effect is most likely the result of a diffraction grating effect. Figure 4 (b) shows evidence of the diffraction grating in measured ASR where a secondary weaker peak at $\lambda / 2$ appear in signal response for the $\lambda>760 \mathrm{~nm}$ cases. The out-of-band response changes the weighting of the band-averaged spectral response and causes a shift in the shape of $\mathrm{R}$ versus peak ASR.

As described, the Independent Calibration is developing an instrument model for CPF that will allow the GLAMR-based absolute calibration to be transferred to orbit. Thus, the ability to collect additional data with GLAMR while doing the absolute radiometric calibration has the advantage of providing additional information for the model development. One such test that has been performed for SOLARIS was multiple collects of approximately two hours of SOLARIS images with GLAMR at a constant radiance and single wavelength. Such scans provide insight in the dominant noise types present in the SOLARIS FPA. The results shown here are for measurements performed at an $850 \mathrm{~nm}$ tuned GLAMR wavelength. A total of 5000 illuminated SOLARIS frames were collected along with 1000 dark frames at 1 frame per second rate. The results shown in Figure 5 give the SOLARIS signal as a function of time for a single spatial row of the FPA and the detector corresponding that having the highest response for the $850 \mathrm{~nm}$ wavelength. Also shown are the GLAMR radiance as reported by the transfer radiometer, and the calculated ASR values as a function of time. The solid red line in all graphs is the moving average of 20 data points (or 20 seconds) to highlight lower frequency effects separate from the higher frequency effects. 


\subsection{GLAMR-Based Uncertainties}

Recent collaborative efforts with the developers of SIRCUS has led to an estimate of the GLAMR absolute radiometric output uncertainty of $0.57 \%(\mathrm{k}=2)$ at $860 \mathrm{~nm}$. The evaluation includes uncertainties originating from the transfer radiometer absolute calibration and stability, transfer of the radiometer calibration to that of the GLAMR sphere, representativeness of the spheres transfer radiometer monitor, and measurement noise. The standard deviation of the sphere radiance during the SOLARIS calibration is shown in Figure 2 (b). From $400 \mathrm{~nm}$ to $1000 \mathrm{~nm}$ range, the standard deviation in measured radiance is centers near $10^{7} \mathrm{~W} / \mathrm{cm}^{2} \mathrm{sr}$. The uncertainty of the GLAMR radiance output is also tested from long-term scans. At $850 \pm 0.2 \mathrm{~nm}$, the GLAMR output radiance is centered around $387.4 \times 10^{6} \mathrm{~W} / \mathrm{cm}^{2} \mathrm{sr}$ and the standard deviation is near $1 \times 10^{6} \mathrm{~W} / \mathrm{cm}^{2} \mathrm{sr}$. The GLAMR integrating sphere spatial uniformity was evaluated by both NIST and NASA and show that sphere non-uniformity contributes $0.14 \%(\mathrm{k}=2)$ uncertainty.
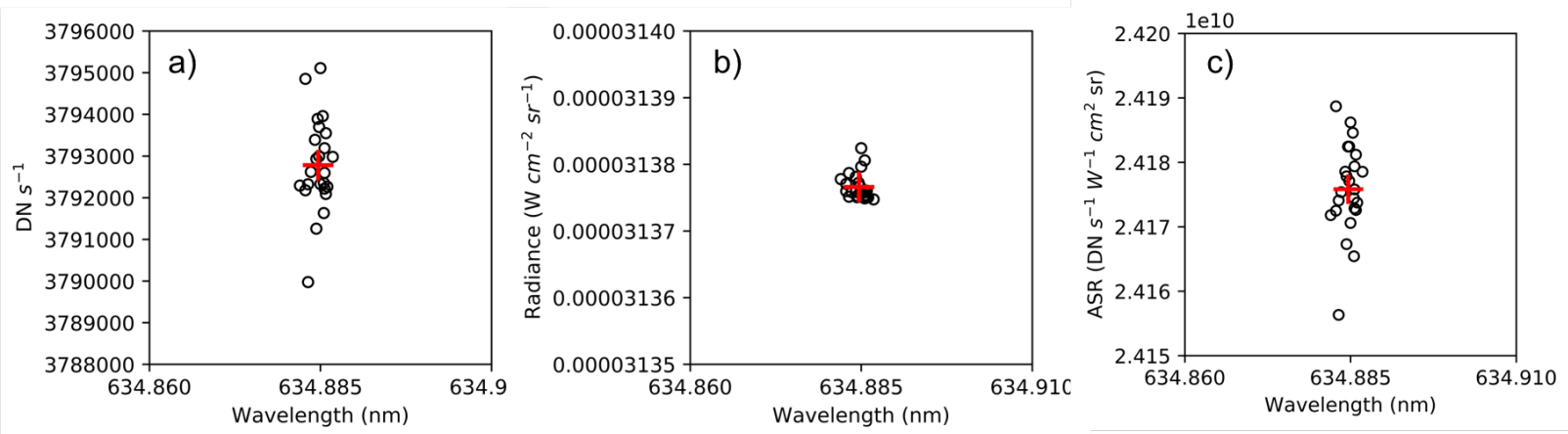

Figure 6. The response of the pixel located at 1250:1320 (vertical:horizontal) is shown for tuned GLAMR wavelength of $634 \mathrm{~nm}$. Each data point is obtained from an individual image, and GLAMR reading.

\subsection{SOLARIS-Based Uncertainties}

Achieving the low levels of absolute uncertainty needed for CPF means that the noise in the calibration system must be far lower than that of the sensor under test. Figures 5 and 6 demonstrate the variability of both the GLAMR source and the SOLARIS signal for coincident collections. The data shown assumes that the noise in the GLAMR transfer radiometers is much smaller than that of the GLAMR source and the radiometer output is representative of the source variability. Evaluation of the SOLARIS output indicates noise at the $1 \%$ a coefficient of variation, or the standard deviation ratio to the mean value, is near $1.25 \%$ while the variation in GLAMR source radiance is near $0.02 \%$. The variation from the GLAMR source is further mitigated by the fact that calculating the ASR values correct for source changes.

Figure 6 shows an example of a data cluster at the $635 \mathrm{~nm}$ GLAMR wavelength for a single spatial row and the spectral detector with peak response at this GLAMR wavelength (1250:1320 (vertical:horizontal)). The peak response of the pixel is at $634.880 \mathrm{~nm}$ wavelength where the averaged signal is $3.793106 \mathrm{DN} / \mathrm{s}$ with the standard deviation of $1.108103 \mathrm{DN} / \mathrm{s}$. The corresponding GLAMR source output at the same wavelength (and time) is $3.138 \times 10^{5} \mathrm{~W} / \mathrm{cm}^{2} \mathrm{sr}$ with a standard deviation of $2.02 \times 10^{9} \mathrm{~W} / \mathrm{cm}^{2} \mathrm{sr}$.

\section{INSTRUMENT LEVEL FIELD TESTING}

The results of the laboratory calibration, and the possible issues related to instrument stability, sensor frame rate, and stray light can be tested using field measurements. ${ }^{16}$ The field results presented here took place at GSFC on July 10th, 2019 between 11:00 am to 11:30 am EDT. The test consisted of SOLARIS and a non-imaging field spectrometer located adjacent to each other viewing vertically downward. A white diffuser panel was used as a uniform target. During measurements, the reflected Solar radiance, as well as the shaded panel and dark current data is collected. Figure 7 (a) shows the results from SOLARIS and field spectrometer instrument, and Figure 7 (b) shows the comparison between two results. The instrument measurement equation is, 


$$
E_{o b j}(\lambda)=S_{o b j}(\lambda) / R(\lambda)
$$

where $E_{o b j}(\lambda)$ is the SI-traceable reflected radiance $\left(\mathrm{W} / \mathrm{m}^{2} \mathrm{srnm}\right)$, and $S_{o b j}(\lambda)$ is the instrument measured scene that is given in instrument data/digital numbers $(\mathrm{DN} / \mathrm{s}), R(\lambda)$ is the band averaged spectral responsivity as obtained in Equation 2 in units of $\mathrm{DNs}^{-1} \mathrm{~W}^{-1} \mathrm{~m}^{2} \mathrm{srnm}$.
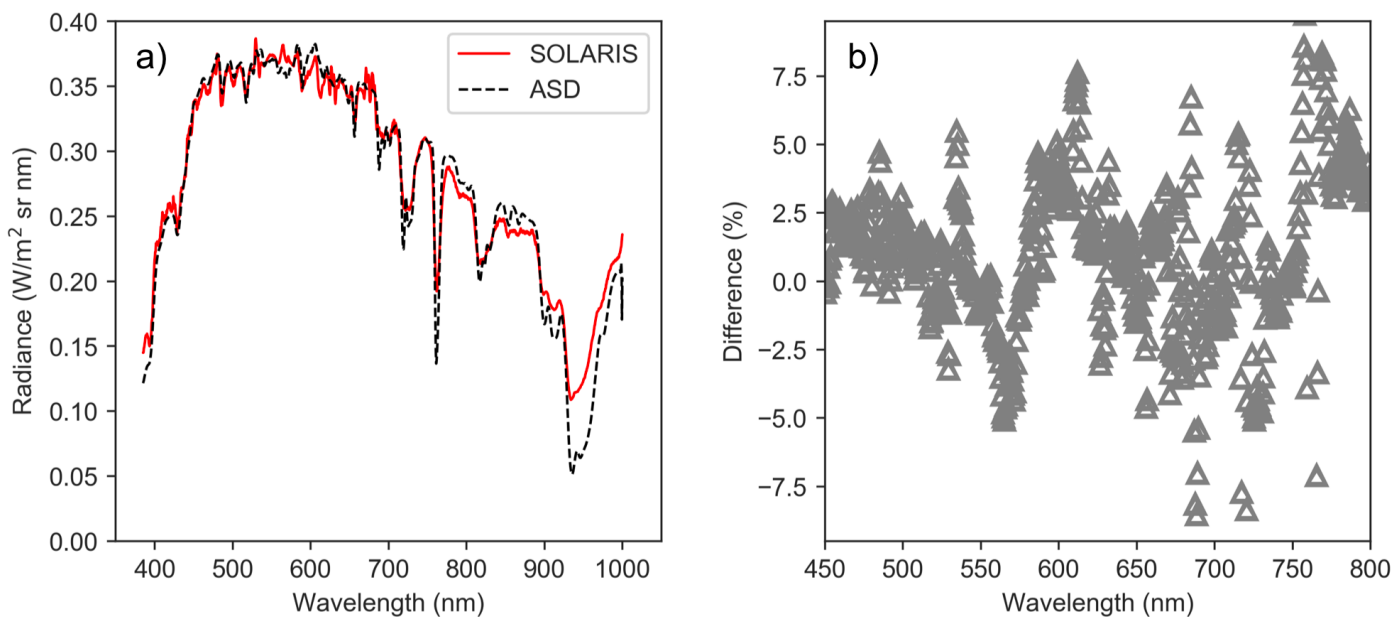

Figure 7. (a) The measurements results of solar reflectance from reflective panel are shown for SOLARIS and field spectrometer instruments. The difference between the field spectrometer and SOLARIS results are presented (b) in percent difference, and (c) in binned histogram formats.

There is approximately $7 \%$ mismatch between the field spectrometer and SOLARIS instruments is observed from $350 \mathrm{~nm}$ to $1000 \mathrm{~nm}$ spectral range. SOLARIS noise level is near $5 \%$ peak-to-peak which is one of the reasons of the measured mismatch in radiance data. field spectrometer related uncertainties, field of view related spatial sampling difference, sky conditions between two consecutive measurements are some of the factors that can disturb the perfect match in results. At the same time, the spectral features of the atmospheric absorption and scattering are detected by the SOLARIS instrument, further measurements of direct Solar and Lunar measurements are anticipated to take place.

\section{CONCLUSION}

The CPF Independent Calibration includes a laboratory based absolute radiometric calibration procedure that relies on detector-based, SI-traceable standards. The laboratory characterization process is performed by utilizing a monochromatic, tuneable, and uniform light source from GLAMR. Developing and testing of calibration protocols for the CPF Independent Calibration are relying on the SOLARIS calibration demonstration system that is an imaging spectrometer similar to that planned for CPF. The SOLARIS calibration approach described here has been used to derive ASR and R for a large subset of the SOLARIS FPA showing the feasibility of GLAMR for the independent calibration. The dominant calibration uncertainty is determined to be originated from the SOLARIS noise, while the GLAMR based uncertainty contribution to ASR values is estimated near $0.2 \%$. Long-term data collections are performed to evaluate GLAMR stability, while periodically collecting SOLARIS images. The GLAMR radiance coefficient of variance is determined $0.02 \%$ from an hour long data collection. Due to the high fidelity in the temporal synchronization during calibration measurements, the uncertainly level of the SOLARIS absolute spectral response is smaller than the raw signal output of SOLARIS.

The band averaged responsivity of SOLARIS is calculated by using the wavelength dependent ASR data. These results later used in processing the Solar reflection measurements taken at the NASA GSFC. The comparison between the field spectrometer and the SOLARIS results show that the overall mismatch is within $5 \%$ from $450 \mathrm{~nm}$ to $800 \mathrm{~nm}$ spectral range. 
In summary, the GLAMR test setup is proven to deliver the absolute radiometric accuracy needed for the $\mathrm{CPF}$ Independent Calibration. The planned calibration approach for CPF has been through the calibration of SOLARIS, including the data acquisition and refinement of the processing methods. The test protocol used in this paper is designed to achieve operational use of GLAMR for the CPF independent calibration that will allow near real-time evaluation of the CPF sensor during testing.

\section{REFERENCES}

[1] Roithmayr, C. M., Lukashin, C., Speth, P. W., Kopp, G., Thome, K., Wielicki, B. A., and Young, D. F., "Clarreo approach for reference intercalibration of reflected solar sensors: On-orbit data matching and sampling," IEEE Transactions on Geoscience and Remote Sensing 52(10), 6762-6774 (2014).

[2] Wielicki, B. A., Young, D. F., Mlynczak, M. G., Thome, K. J., Leroy, S., Corliss, J., Anderson, J. G., Ao, C. O., Bantges, R., Best, F., Bowman, K., Brindley, H., Butler, J. J., Collins, W., Dykema, J. A., Doelling, D. R., Feldman, D. R., Fox, N., Huang, X., Holz, R., Huang, Y., Jin, Z., Jennings, D., Johnson, D. G., Jucks, K., Kato, S., Kirk-Davidoff, D. B., Knuteson, R., Kopp, G., Kratz, D. P., Liu, X., Lukashin, C., Mannucci, A. J., Phojanamongkolkij, N., Pilewskie, P., Ramaswamy, V., Revercomb, H., Rice, J., Roberts, Y., Roithmayr, C. M., Rose, F., Sandford, S., Shirley, E. L., Smith, W. L., Soden, B., Speth, P. W., Sun, W., Taylor, P. C., Tobin, D., and Xiong, X., "Achieving climate change absolute accuracy in orbit," Bull. Amer. Meteor. Soc. 94(10), 1519-1539 (2013).

[3] McAndrew, B., McCorkel, J., Shuman, T., Zukowski, B., Traore, A., Rodriguez, M., Brown, S., and Woodward, J., "Goddard laser for absolute measurement of radiance for instrument calibration in the ultraviolet to short wave infrared," in [2018 Conference on Lasers and Electro-Optics (CLEO)], 1-2, IEEE (2018).

[4] Oudrari, H., McIntire, J., Xiong, X., Butler, J., Ji, Q., Schwarting, T., Lee, S., and Efremova, B., "Jpss-1 viirs radiometric characterization and calibration based on pre-launch testing," Remote Sensing 8(1), 41 (2016).

[5] Oudrari, H., McIntire, J., Xiong, X., Butler, J., Ji, Q., Schwarting, T., and Angal, A., "An overall assessment of jpss-2 viirs radiometric performance based on pre-launch testing," Remote Sensing 10(12), 1921 (2018).

[6] Moyer, D., McIntire, J., Young, J., McCarthy, J. K., Waluschka, E., Xiong, X., and De Luccia, F. J., "Jpss1viirs prelaunch polarization testing and performance," IEEE Transactions on Geoscience and Remote Sensing 55(5), 2463-2476 (2017).

[7] Angal, A., McCorkel, J., Cook, B., Thome, K., et al., "Radiometric calibration of g-liht's imaging spectrometer using glamr for satellite sensor intercalibration," in [Earth Observing Systems XX], 9607, 96070C, International Society for Optics and Photonics (2015).

[8] Thompson, P. L. and Hill, P. C., "Conceptual optical design and system engineering of the clarreo/rs (reflected solar) instrument suite," in [Imaging Spectrometry XVII], 8515, 85150N, International Society for Optics and Photonics (2012).

[9] Thome, K., McCorkel, J., and McAndrew, B., "Demonstrating the error budget for the climate absolute radiance and refractivity observatory through solar irradiance measurements," in [Earth Observing Systems $X X]$, 9607, 96071C, International Society for Optics and Photonics (2015).

[10] Thome, K., McCorkel, J., and McAndrew, B., "Error budget for a calibration demonstration system for the reflected solar instrument for the climate absolute radiance and refractivity observatory," in [Imaging Spectrometry XVIII], 8870, 887008, International Society for Optics and Photonics (2013).

[11] Anderson, N., Thome, K., Czapla-Myers, J., and Biggar, S., "Design of an ultra-portable field transfer radiometer supporting automated vicarious calibration," in [Earth Observing Systems XX], 9607, 960709, International Society for Optics and Photonics (2015).

[12] Butler, J. J., Johnson, B. C., Brown, S. W., Yoon, H. W., Barnes, R. A., Markham, B. L., Biggar, S. F., Zalewski, E. F., Spyak, P. R., Cooper, J. W., et al., "Radiometric measurement comparisons using transfer radiometers in support of the calibration of nasa's earth observing system (eos) sensors," in [Sensors, Systems, and Next-Generation Satellites III], 3870, 180-193, International Society for Optics and Photonics (1999). 
[13] Thome, K., McCorkel, J., Hair, J., McAndrew, B., Daw, A., Jennings, D., and Rabin, D., "Test plan for a calibration demonstration system for the reflected solar instrument for the climate absolute radiance and refractivity observatory," in [Remote Sensing System Engineering IV], 8516, 851602, International Society for Optics and Photonics (2012).

[14] McCorkel, J., Thome, K., and Lockwood, R. B., "Absolute radiometric calibration of narrow-swath imaging sensors with reference to non-coincident wide-swath sensors," IEEE Transactions on Geoscience and Remote Sensing 51(3), 1309-1318 (2013).

[15] Barnes, R. A., Brown, S. W., Lykke, K. R., Guenther, B., Xiong, X., and Butler, J. J., "Comparison of two methodologies for calibrating satellite instruments in the visible and near infrared," in [Earth Observing Missions and Sensors: Development, Implementation, and Characterization], 7862, 78620C, International Society for Optics and Photonics (2010).

[16] Angal, A., McCorkel, J., and Thome, K., "Evaluation of glamr-based calibration for si-traceable field reflectance retrievals," in [Earth Observing Systems XXI], 9972, 99721U, International Society for Optics and Photonics (2016). 